\title{
OGSE absolute radiometric calibration in VIS and NIR
}

Sonia Valls, Maxime Ranchin, Sabine Balliion, Wilfried Glastre, Yannig Durand

Sonia Valls, Maxime Ranchin, Sabine Balliion, Wilfried Glastre, Yannig Durand, "OGSE absolute radiometric calibration in VIS and NIR," Proc. SPIE 11852, International Conference on Space Optics — ICSO 2020, 118524B (11 June 2021); doi: 10.1117/12.2599650

SPIE Event: International Conference on Space Optics - ICSO 2021, 2021, Online Only 


\section{International Conference on Space Optics-ICSO 2020}

Virtual Conference

30 March-2 April 2021

Edited by Bruno Cugny, Zoran Sodnik, and Nikos Karafolas
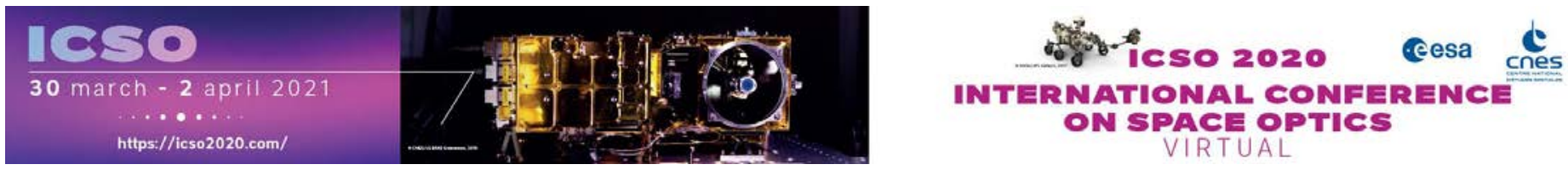

\section{OGSE absolute radiometric calibration in VIS and NIR}

\section{eesa isso procedilings denes}




\title{
OGSE absolute radiometric calibration in VIS and NIR
}

\author{
Sonia Valls ${ }^{\mathrm{a}}$, Maxime Ranchin ${ }^{\mathrm{a}}$, Sabine Ballion ${ }^{\mathrm{a}}$, Wilfried Glastre ${ }^{\mathrm{b}}$, Yannig Durand ${ }^{\mathrm{c}}$ \\ ${ }^{a}$ Thales Alenia Space, 5 Allée des Gabians, B.P. 99, 06150 Cannes la Bocca, France \\ ${ }^{\mathrm{b}}$ Bertin Technologies, 155 Rue Louis Armand, 13290 Aix-En-Provence, France \\ ${ }^{c}$ European Space Agency, ESTEC, P.O. Box 299, 2200 AG Noordwijk, The Netherlands
}

\begin{abstract}
For Meteosat Third Generation (MTG), the new generation of geostationary meteorology satellites, the Flexible Combined Imager (FCI) instrument will bring a new step in image accuracy. The FCI is an imaging radiometer which will be able to provide Earth images with unprecedented absolute radiometric levels in its whole working spectrum, ranging from visible, near infrared to infrared.
\end{abstract}

ESA has chosen Thales Alenia Space as MTG Prime contractor and responsible for the Assembly Integration and Test of the FCI instrument. The absolute radiometric calibration of the instrument needs to be better than 3\%, an absolute accuracy level never reached before.

To achieve this challenge, Thales Alenia Space has developed, along with Bertin Technologies, a new Optical Ground Support Equipment (OGSE) dedicated to FCI tests and calibration, which is called MOTA : Multi Optical Test Assembly.

Thales Alenia Space has developed a rigorous metrology chain from metrology primary standard to the instrument detection chain, passing through several steps, to ensure absolute radiometric calibration of the FCI instrument.

Thales Alenia Space has a long history of experience in radiometric tests and metrology, and this calibration method lies on all our previous experiences gathered in the achievement of numerous programs.

This paper will describe historical calibration method, and then explain all the optimisations and evolutions brought by the new method, regarding secondary standard, spectro-radiometric monitoring, environment management, and a focus will be made on importance of the hardware quality.

Note: This work is performed under an ESA contract to Thales Alenia Space.

Keywords: OGSE, radiometry, absolute calibration, spectro-radiometer, secondary standard, monitoring 


\section{CONTEXT}

\subsection{Past experience and traditional method}

Thales Alenia Space has a long experience in Assembly Integration and Test of Optical and Observation instrument of many types : High Resolution imagers, Spectro-radiometers, radiometer imagers, sounders...

For each type of instrument, and each specific mission, radiometric calibration is done on-ground to validate the performances of the instrument before launch. The calibration accuracy depends on the instrument type and the mission requirements. Usual absolute calibration level is about $5 \%$ to $8 \%$ at 3 sigma. In order to achieve this performance, traditional method of direct calibration is used.

The direct calibration method consists in a metrology chain as follows :

1. Primary standard in national metrological laboratory is the absolute reference

2. The spectro-radiometer is a secondary standard, periodically sent to metrological laboratory for calibration with respect to primary standard

3. The OGSE, that will be used as a calibration source in front of the flight instrument, is calibrated with respect to the spectro-radiometer

4. The OGSE radiance is followed by monitoring, usually broadband photodiode.

This method has the advantage to be direct and easy to apply. Indeed, the spectro-radiometer is calibrated every one to two years, depending on its long terms stability, and is then available to be used at any time between two periodic calibrations.

The drawback of this method is the performance limitation. Usual absolute calibration measurement capabilities of metrological laboratories allow to reach about 5 to $6 \%$ uncertainty ( 3 sigma) for detector calibration in visible spectrum.

This is why, in order to improve absolute radiometric calibration accuracy, Thales Alenia Space has developed a new method to be implemented on FCI instrument, in order to reach the 3\% (3 sigma) requirement.

\subsection{MOTA description}

For the MTG program, Thales Alenia Space has developed, along with Bertin Technologies, a new Optical Ground Support Equipment dedicated to FCI tests and calibration, which is called MOTA : Multi Optical Test Assembly as shown on Figure 1.
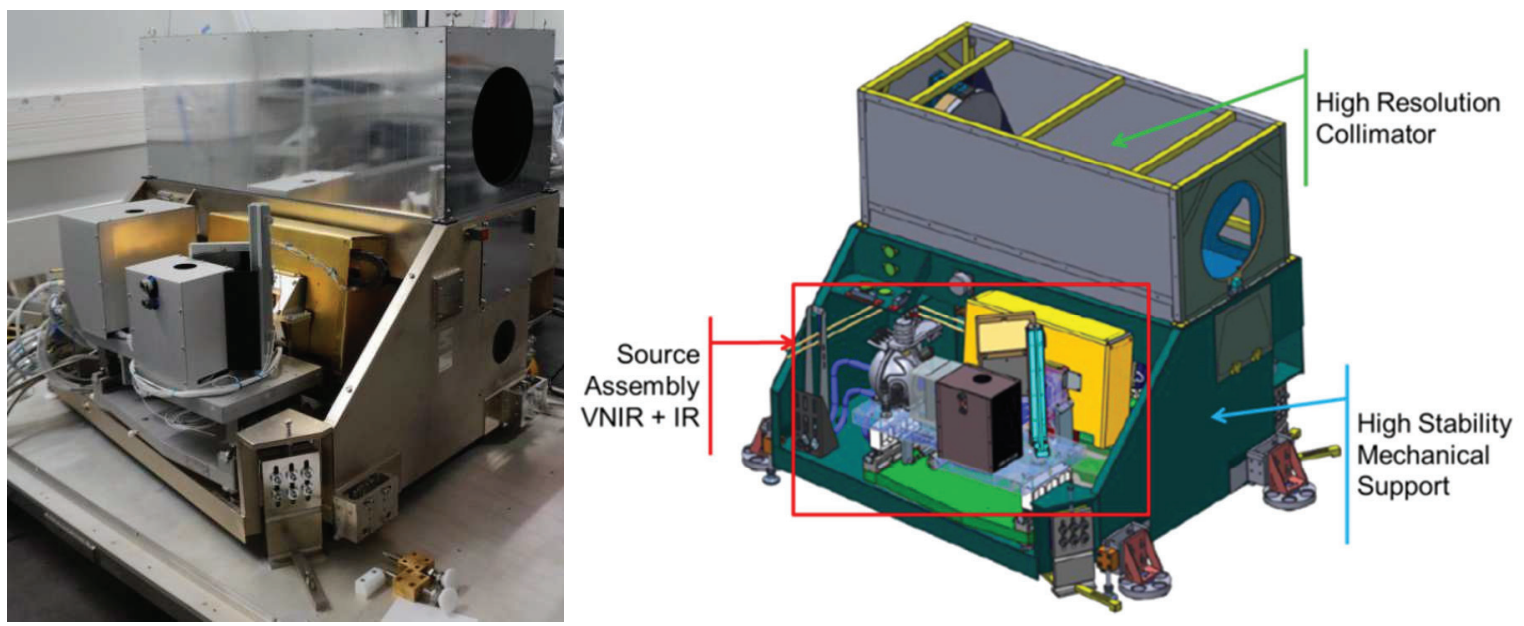

Figure 1 : Picture of MOTA : Multi Optical Test Assembly ; CAD view with description of sub-assemblies 
The MOTA allows to simulate objects at infinite, and is composed of a mechanical structure supporting a collimator and a source assembly. The measurements performed with the MOTA allow to adjust the FCI and to characterize all its optical and radiometric performances in visible and NIR spectra: optical adjustment, MTF characterisation, coregistration, straylight, radiometric relative and absolute calibrations. MOTA is then a complex OGSE that answers to many accuracy requirements in a wide spectrum of interest (440 to $2250 \mathrm{~nm}$ for radiometry, and to $14.1 \mu \mathrm{m}$ for optical and straylight tests), on a large radiance dynamic (2 orders of magnitude), with high spectral resolution and under vacuum conditions. Detailed description of MOTA design and performance are presented in [1].

Bertin Technologies proposed and developed a calibration method for MOTA [2], under Thales Alenia Space supervision and approval. After MOTA delivery, Thales Alenia Space has worked to improve further the method.

\section{SECONDARY STANDARD AND TRANSFER METHOD}

\subsection{Transfer method}

The calibration method proposed by Bertin Technologies during MOTA development is presented in details in [2]. It is based on the transfer method and can be summarised in the following steps and is illustrated on Figure 2:

1. Primary standard source in national metrological laboratory is the absolute reference

2. Portable Secondary standard source is periodically sent for calibration to metrological laboratory for calibration with respect to primary standard

3. The OGSE source, that will be used as a calibration source in front of the flight instrument, is calibrated : the calibration is transferred from the secondary standard to the OGSE using a spectro-radiometer (Figure 2)

4. The OGSE radiance is followed by monitoring, usually broadband photodiode

This method requires a secondary standard source, in addition to the spectro-radiometer. This is an initial investment, that allows to run a calibration process that always compares a source to another. This is why a better performance can be reached.
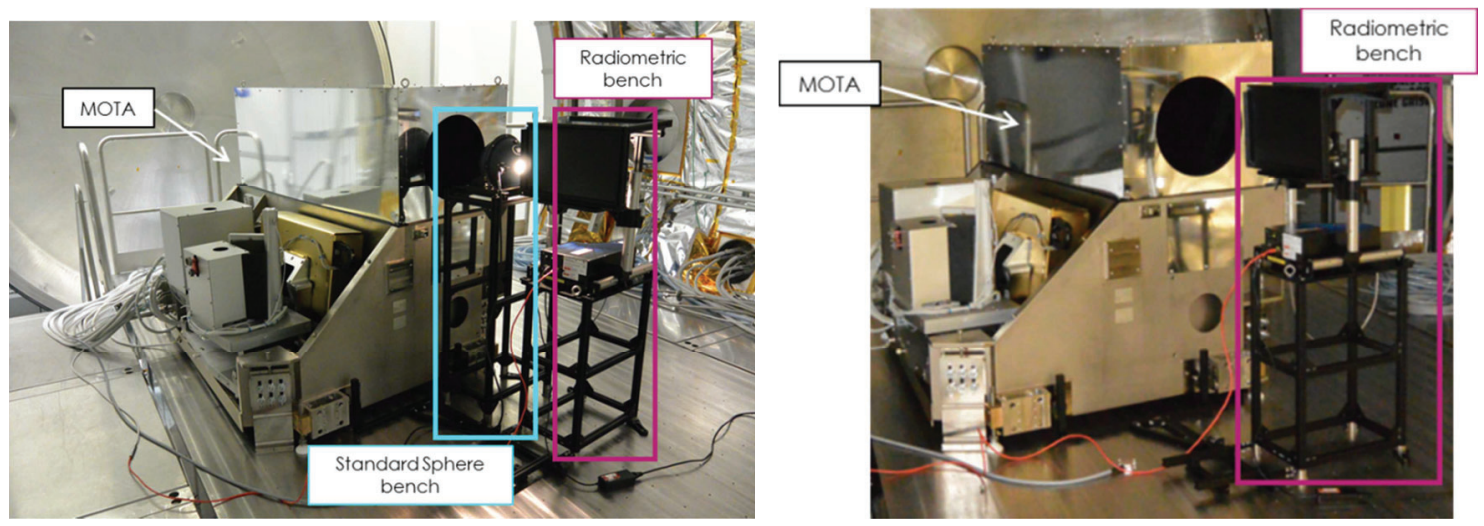

Figure 2 : Transfer method : step 1 - spectro-radiometer in front of the secondary standard sphere ; step 2 - spectroradiometer in front of MOTA OGSE. The spectro-radiometer configuration has not changed between both steps.

The secondary standard source is used only during the timeslot of the transfer of calibration to the OGSE to minimise the operation duration. Indeed, the calibration is usually ensured by the metrology laboratories for a duration between 50 to 100 hours of operations. Then secondary standard source needs to be recalibrated. Moreover, even if it is not operated, the secondary standard source has to be recalibrated about every 6 months due to potential drifts.

The calibration is passed through OGSE source using a highly stable spectro-radiometer, with a detector thermally controlled, and a good spectral resolution (2.5 nm in VIS, $10 \mathrm{~nm}$ in NIR1 and $7 \mathrm{~nm}$ in NIR2 regions, see $\S 3.2)$. 
The advantage of the transfer method is that it allows to operate a spectro-radiometer that does not require to be calibrated. The measurements on secondary standard and on the OGSE are done sequentially without changing the operational configuration of the spectro-radiometer. This allows to remove most accuracy budget contributors coming from the spectro-radiometer : spectro calibration, reproducibility of connexions. In the end, the remaining main contributor is the spectro-radiometer measurement repeatability which is quite good (between 0.1 and $0.2 \%$ ).

\subsection{Choice of secondary standard}

In the transfer method, the most critical hardware is then the secondary standard source. Two types of sources have been considered : an integrating sphere, and a FEL lamp associated to a diffuser.

The FEL lamp and diffuser solution, which are historical tools, impose some practical constraints : precise distances and angles in the configuration, calibrations in two steps (one for the lamp, one for the diffuser), power of the source not adjustable to the need (specific dynamic to be reached for FCI instrument use case). This solution is not robust to frequent transports, because of critical need of alignment reproducibility between lamp and diffuser. Moreover, the management of straylight of such configuration is quite a challenge.

The integrating sphere solution allows more flexibility and adaptability to the need. It is robust to transport, often required for secondary standard, (every 50 hours of operation or every 6 months). The dimensions, power and configuration of use offer possible adjustments. The straylight of the configuration can be managed by usual baffles. Finally, the output port can be closed to protect the hardware from contamination, which can be an issue on a cleanroom environment.

Consequently, the chosen secondary standard was an integrating sphere. Bertin Technologies chose to procure a sphere similar to the one developed for the source assembly of MOTA, see Figure 3, for which uniformity performance was already demonstrated. It was associated to a high performance power supply stabilised down to $+/-0.06 \%$ (at $3 \sigma$ ). This ensures that radiance variations $\&$ drifts due to power supply is less than $0,37 \%$ at $3 \sigma$ for all channels.

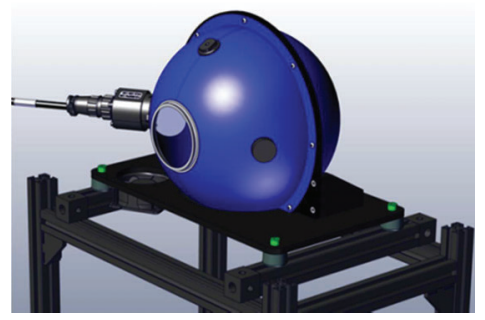

Figure 3 : CAD view of secondary standard integrating sphere provided by Bertin Technologies

After primary runs of calibration procedure, Thales Alenia Space noticed that the radiance level of the secondary standard sphere was unique and quite high compared to the possible radiance levels at the output of the MOTA. The transfer method measurement is done with the same configuration of the spectro-radiometer in front of the secondary standard and in front of the OGSE. Thales Alenia Space decided then to procure a new secondary standard (see Figure 4) with variable radiance in order to realise the transfer method measurements with several levels of interest, similar between the secondary standard and the MOTA levels. This allows to avoid possible biases of configuration during the transfer measurements. 


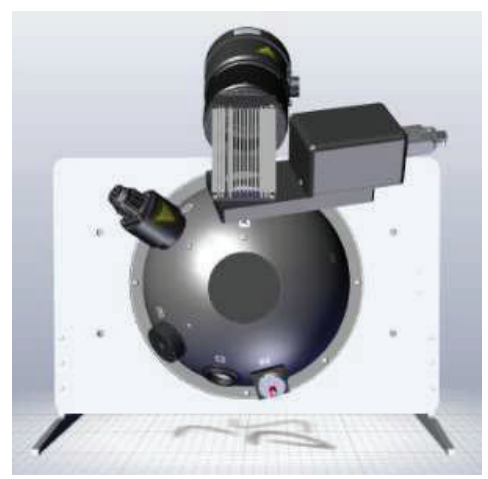

Figure 4 : Picture of secondary standard provided by Thales Alenia Space, with internal lamp and external variable lamp

\subsection{Comparison with traditional method}

In the traditional method using the calibrated spectro-radiometer, the advantage is that the secondary standard is a device that can be used during 1 to 2 years before needing recalibration. This method is easier and more direct to implement. However, the absolute calibration accuracy ensured by the metrological laboratories for spectro-radiometer is limited to a value about $5 \%(3 \sigma)$.

The transfer method require more hardware : a secondary standard source in addition to the spectro-radiometer. The calibration of the secondary standard is valid during a limited operating time, usually about 50 hours of operation or 6 months even without operations. This method is also more complex to implement, because the levels of the secondary standard need to be mastered and chosen close to the levels seen from the OGSE. On the other hand, this method allows to reach better accuracy of absolute calibration. The Calibration Measurement Capabilities (CMC) of metrological laboratories are about $1.5 \%$ to $2.5 \%(3 \sigma)$ for visible and NIR wavelengths of MTG (between 440 and $2250 \mathrm{~nm}$ ).

\section{SPECTRO-RADIOMETRIC MONITORING}

\subsection{Optimisations brought by spectro-radiometric monitoring}

One of the major improvement brought by the calibration process settled by Bertin Technologies for MOTA is the monitoring of MOTA source. Traditionally, the monitoring of the OGSE sources is done with a photodiode, as previously mentioned (step 4 of calibration methods $\$ 1.1 \& \$ 2.1$ ). This kind of monitoring provides one value of monitoring that corresponds to the integrated radiance over the full sensitive spectrum of the photodiode.

This broadband monitoring does not detect drifts in colour temperature, that can be caused by lamps ageing, or local variations of the spectral radiance curve, caused for example by water vapour absorption. Yet, the requirements on FCI concern spectral radiance of each narrow channel of the instrument, see channels characteristics in Table 1.

Table 1. Spectral channel of FCI for VIS and NIR bands

\begin{tabular}{|c|c|c|}
\hline Channel name & central wavelength (nm) & Spectral bandwidth (nm) \\
\hline VIS0.4 & 444 & 60 \\
\hline VIS0.5 & 510 & 40 \\
\hline VIS0.6 & 640 & 50 \\
\hline VIS0.8 & 865 & 50 \\
\hline VIS0.9 & 914 & 20 \\
\hline NIR1.3 & 1380 & 30 \\
\hline NIR1.6 & 1610 & 50 \\
\hline NIR2.2 & 2250 & 50 \\
\hline
\end{tabular}


Bertin Technologies chose to realise MOTA monitoring using the spectro-radiometer itself. This is then a breakthrough with regards to previous methods which relied on radiometry source with a simpler monitoring based on photodiode.

Consequently, the MOTA monitoring has a spectro-radiometric extension covering the whole spectrum (from $0.4 \mu \mathrm{m}$ to $2.25 \mu \mathrm{m}$ ) with a large dynamic range (radiance is calibrated at all levels from minimal to maximum radiance).

The spectro-radiometer used for the transfer method, to calibrate spectral radiance at the output of the MOTA, is the same for monitoring of the MOTA during the whole test sequence in front of the FCI. The spectral monitoring of MOTA is realised with a fibered link between MOTA source (integrating sphere inside the source assembly), and the spectroradiometer. The fibre link allows the use of MOTA under vacuum for tests sequence on FCI (see Figure 5).

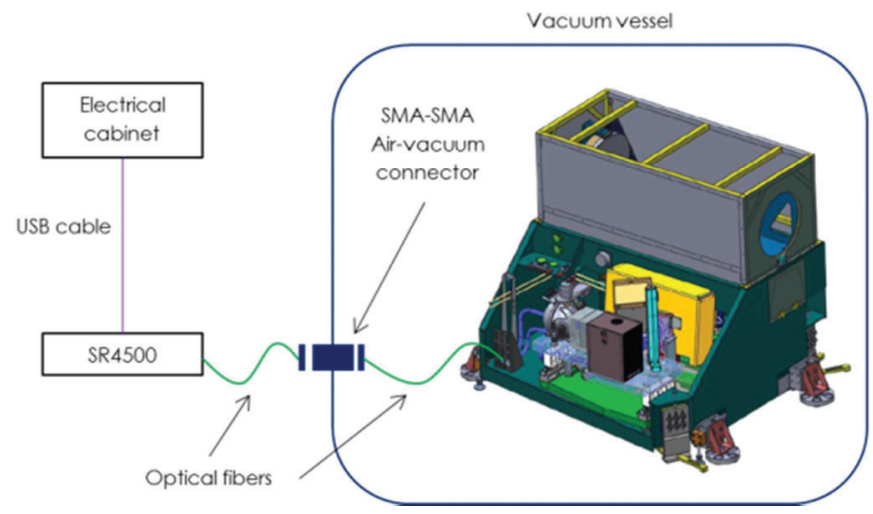

Figure 5 : MOTA monitoring configuration, compatible with vacuum test sequence

\subsection{Choice of the spectro-radiometer}

After study of available products on the market, Bertin Technologies selected the SR4500 spectrometer from Spectral Evolution (see Figure 6), showing a high stability over the total 400-2500 $\mathrm{nm}$ spectral range, low straylight resulting in reduced cross-talk between wavelengths and high sensitivity to cover the lower parts of the dynamic.

It is designed with no moving part, and based on 3 high performances cooled linear detectors : VIS (350-1000 nm), NIR1 (1000-1900nm) and NIR2 (1900-2500nm). This provides stability down to $0.3 \%$ over several months. [1]

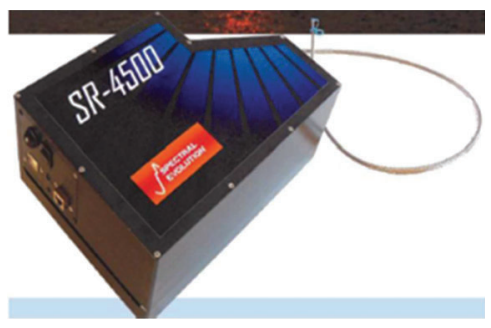

Figure 6 : SR4500 chosen spectro-radiometer

For the transfer method, the measurements configuration must remain the same between measurement on secondary standard and on OGSE. Consequently, Bertin Techologies provided a radiometric bench constituted of a radiance head, a bundle of fibres and a mechanical support. The radiance head consists of an entrance diaphragm, a parabolic mirror, and the entrance of the bundle of fibres at the focal point of the mirror (see Figure 7). The bundle of fibres has a square shape and allows to collect the flux from all the MOTA square field of view. The entrance diaphragm limits the incoming beam diameter to avoid straylight, along with mat black baffles all around the radiance head. 


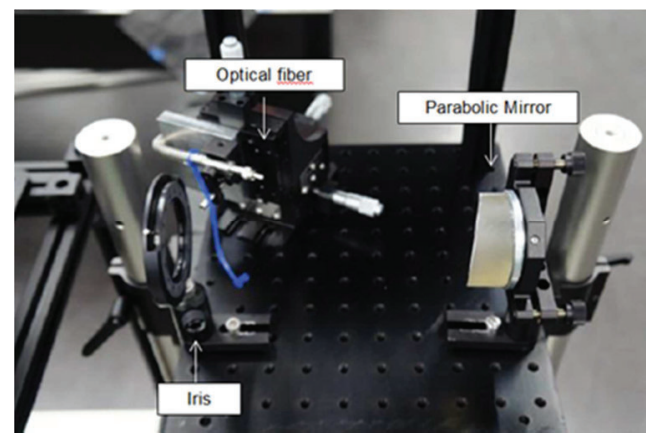

Figure 7 : Custom SR4500 radiance head provided by Bertin Technologies, for transfer method measurements.

\section{ENVIRONMENT MANAGEMENT}

\subsection{Vacuum environment}

The initial radiometric calibration (described in \$2) of MOTA has to be run under air environment because the spectroradiometer body cannot be vacuum compatible and has to be close to the source (secondary standard or OGSE) for this measurement : the bundle of fibres must remain short to maximise the collected flux. However, the final performances of the FCI instrument must be measured in its representative environment which is vacuum. Consequently, the air to vacuum transition has to be integrated in the radiometric calibration process of the MOTA.

Bertin Technologies proposed a method of air to vacuum transition management. It is described in details in [2] and can be summarised as follows: the impact of air to vacuum transition is mainly due to water vapour absorption of some wavelengths and then transmission of the optical path. In the case of MOTA VIS and NIR calibration, the impacted channel is $1.38 \mu \mathrm{m}$. The radiometric calibration procedure includes a measurement of relative hygrometry in the configuration at two steps :

1. Secondary standard calibration at metrological laboratory

2. Transfer measurement between secondary standard and OGSE at Thales Alenia Space

A correction factor has been implemented by Bertin Technologies in the post-processing in order to erase the impact of water vapour absorption for the MOTA calibration at $1.38 \mu \mathrm{m}$ channel.

Beside this hygrometry correction, after the absolute calibration is realised, the MOTA radiance is monitored with the spectro-radiometer. Such monitoring allows to take into account the variations due to transition of MOTA configuration from air to vacuum due to other factors like change of lamp colour temperature.

\subsection{Air environment}

In addition to the absolute calibration, additional measurements are required in FCI test sequence for relative radiometric performances. These measurements are run under air environment. To manage this test configuration, Thales Alenia Space has developed an additional post-processing step dedicated to air environment.

The relative hygrometry is measured in the test configuration of MOTA in front of FCI instrument and a correction factor is calculated to compensate the water vapour absorption effect. Indeed, with the knowledge of the relative hygrometry, the transmission along the optical path of about $10 \mathrm{~m}$ inside MOTA and FCI can be deduced. With the initial absolute calibration, the accurate spectro-radiometric monitoring, and the transmission calculation, the radiance level entering the FCI instrument is accurately controlled. Several runs of radiometric relative measurement can then be compared independently from the environment situation and evolution at the times of the measurements. 


\section{IMPORTANCE OF THE HARDWARE QUALITY}

\subsection{Straylight management}

During the commissioning of MOTA by Thales Alenia Space AIT team, all the performances and operational aspects are rigorously reviewed and tested in order to ensure that they are properly managed by the operators for the coming test sequence in front of the instrument.

Several optimisations and modifications of operational aspect were required during this phase. Indeed, the configuration management is a driver for tests, and in the end, may have an impact on performances.

For radiometry configuration, all the light paths must be managed and checked, and the straylight must be reduced as much as possible. Consequently, baffles are usually required. Baffling in the source assembly of the MOTA were not sufficient, and additional baffling has been worked again together by Thales Alenia Space and Bertin Technologies to achieve an optimised configuration as shown on Figure 8.
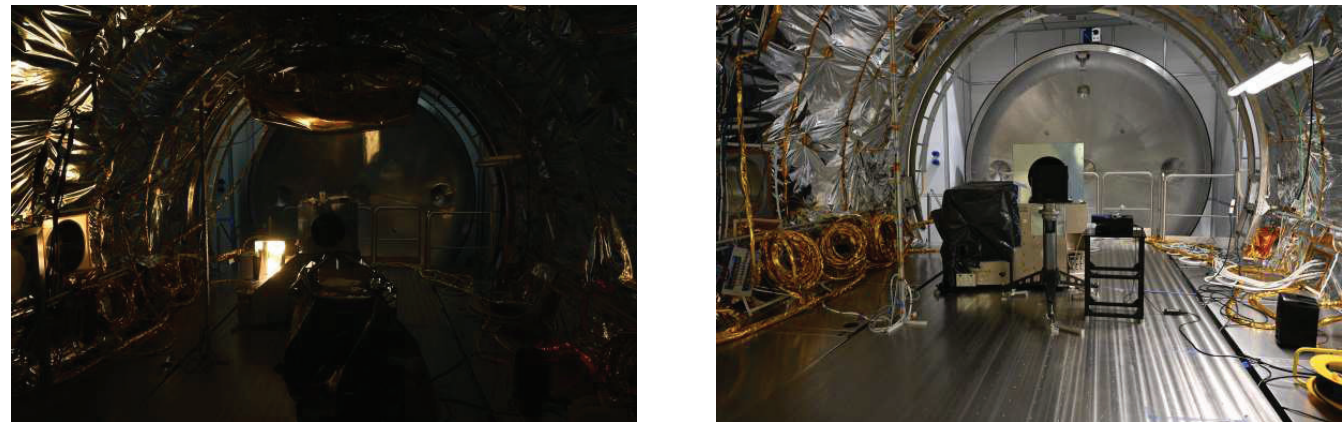

Figure 8 : MOTA calibration configuration before and after source assembly baffles optimisation

Moreover, the baffles around the radiance head were not totally tight and some repeatability issues have been encountered, depending on the environment. A new baffle has been realised by Thales Alenia Space, with a hood shape, to allow only nominal light path through the entrance diaphragm. Former and new baffles are shown on Figure 9.
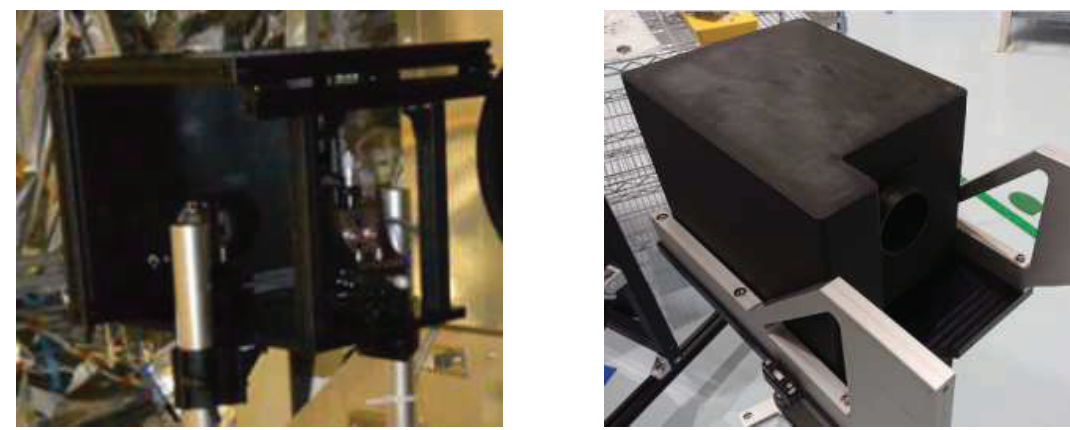

Figure 9 : Radiometric calibration bench optimisation : former baffle and new baffle

\subsection{Robustness and repeatability}

Measurement configuration requires good repeatability in order to reach the very good accuracy that we seek for MOTA calibration. 
Distances and angle, for example between source and detector, has to be well mastered and reproducible. Consequently, the alignments of the configurations are critical. All the steps are run with fine adjustment capabilities and then blocking functions :

- Spectro-radiometer radiance head internal alignment is done with 3D CMM

- Alignment of secondary standard in front of spectro-radiometer is done by mechanical mounting

- Alignment of spectro-radiometer in front of MOTA is done by mechanical wedge

The secondary standard integrating sphere is now on the same support as the radiance head, so the alignment of spectroradiometer in front of secondary standard is highly repeatable around $0.1 \mathrm{~mm}$ (see Figure 10 ).

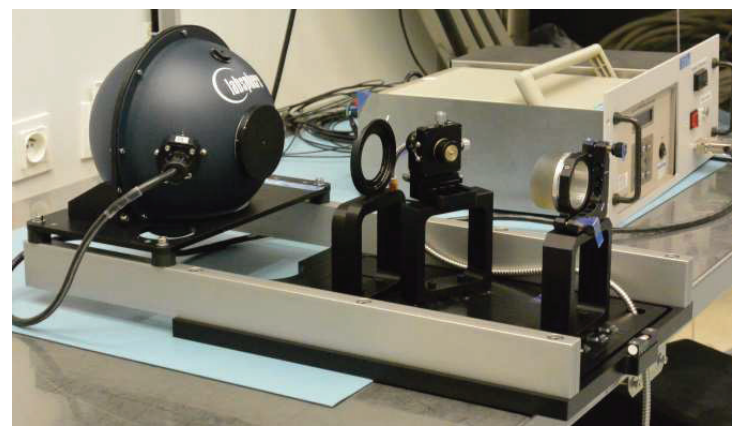

Figure 10: Radiance head and secondary standard on the same support

Repeatability can also be impacted by the fibres connections. Depending on the connector type, it can reach about $1 \%$. Consequently, minimal number of fibres connections and disconnections is realised during the calibration process and then no disconnection is done during the whole monitoring of the test sequence.

The robustness of the configuration is an important aspect for configuration measurements that are settled and dismounted several times. The transfer method calibration has to be done several times on MOTA during one FCI test sequence, and there are four FCI models. The robustness has then been optimised for radiometric calibration bench carrying: the secondary standard integrating sphere, the radiance head and the spectro-radiometer (see Figure 11). The updated structure is no more subject to misalignments when dismounted, transported and re-mounted. It is also heavier, which is a constrain for the operators, but allows a better stability.
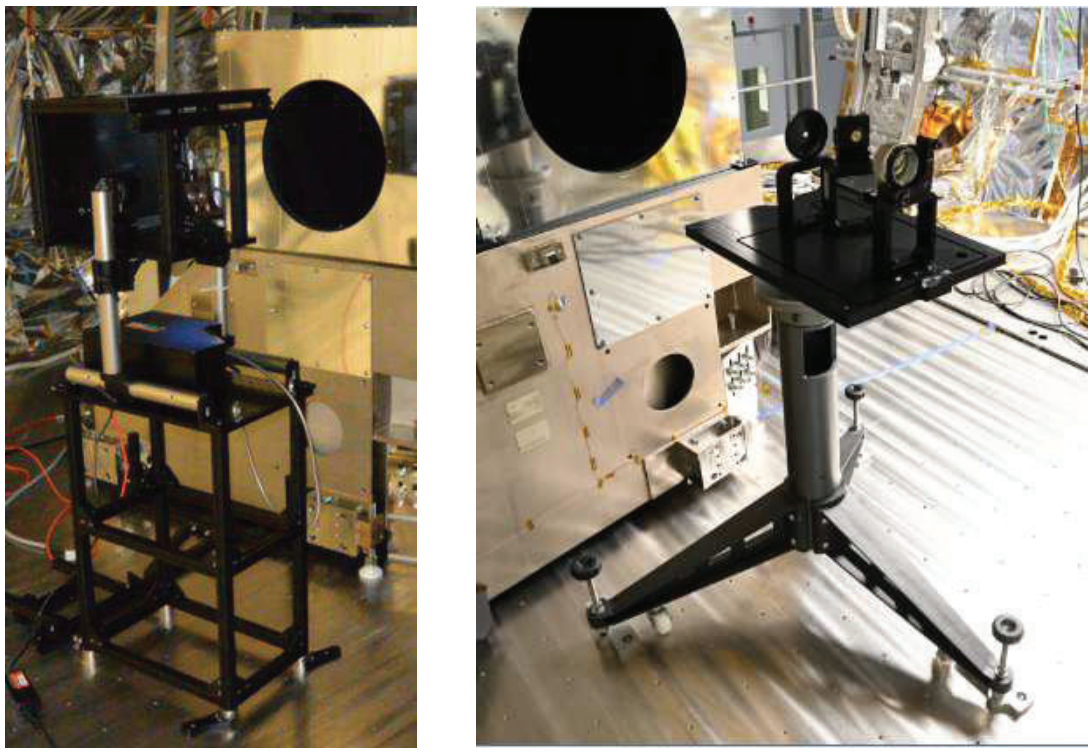

Figure 11 : Radiometric calibration bench (1) initial bench less reproducible (2) optimised bench with improved repeatability and adjustability 


\section{CONCLUSION}

The level of radiometric calibration accuracy required for MTG program lead Thales Alenia Space and Bertin Technologies to innovate the calibration method, bringing a breakthrough over the traditional methods.

The transfer calibration method allows to reach a better level of accuracy, but is a more complex method that requires to manage all the calibration chain. First, the steps of the method were precisely defined. Then, the hardware was carefully chosen: secondary standard integrating sphere, spectro-radiometer with its radiance head, optical fibres and supports. Moreover, the environment of test, ambient and vacuum, was properly taken into account in the method. Finally, the configuration was refined iteratively, to reach a low level of straylight, an accurate repeatability, and a good robustness.

The MOTA radiometric calibration also includes a spectro-radiometric monitoring that follows the behaviour of the source during the entire test sequence, including transition of configuration from air to vacuum and lamps ageing.

The MOTA and its absolute radiometric calibration process are now ready to calibrate the four FCI models.

\section{ACKNOWLEDGEMENT}

The authors would like to thank the PTB, NPL, NIST and LNE for fruitful discussions during this work.

\section{REFERENCES}

[1] E. Compain, et al., "VNIR, MWIR, and LWIR source assemblies for optical quality testing and spectroradiometric calibration of earth observation satellites", Proc. of Optical Design and Engineering VI, vol. 9626, 962626 (2015)

[2] W. Glastre, et al., "Description and performance of the OGSE for VNIR absolute spectroradiometric calibration of MTG-I satellites", Proc. of SPIE, Vol. 10562, 105624H (2017). 